\section{Involvement of the Cerebellum in Parkinson Disease and Dementia with Lewy Bodies}

\author{
Kay Seidel, PhD, ${ }^{1}$ Mohamed Bouzrou, ${ }^{1}$ \\ Nina Heidemann, ${ }^{1}$ Rejko Krüger, MD, ${ }^{2}$ \\ Ludger Schöls, MD, ${ }^{3}$ \\ Wilfred F. A. den Dunnen, MD, ${ }^{4}$ \\ Horst-Werner Korf, MD, ${ }^{1}$ and Udo Rüb, MD ${ }^{1}$
}

Brains from patients with Parkinson disease or dementia with Lewy bodies show aggregation of alpha-synuclein in precerebellar brainstem structures. Furthermore, patients exhibit resting tremor, unstable gait, and impaired balance, which may be associated with cerebellar dysfunction. Therefore, we screened the cerebella of 12 patients with alpha-synucleinopathies for neuropathological changes. Cerebellar nuclei and neighboring white matter displayed numerous aggregates, whereas lobules were mildly affected. Cerebellar aggregation pathology may suggest a prionlike spread originating from affected precerebellar structures, and the high homogeneity between patients with dementia with Lewy bodies and Parkinson disease shows that both diseases likely belong to the same neuropathological spectrum.

ANN NEUROL 2017;81:898-903

$\mathrm{P}$ arkinson disease (PD) and dementia with Lewy bodies (DLB) belong to the human alpha-synucleinopathies, which are characterized by neurodegeneration and widespread aggregation of the alpha-synuclein protein. ${ }^{1-3}$ They were originally described as different diseases, but are more recently considered to represent different clinical patterns within the same clinicopathological spectrum. ${ }^{4,5}$

The disease symptoms of PD include rigidity, bradykinesia, gait and postural instability, resting tremor, impaired balance and postural reflexes, and a pronounced L-dopa responsiveness of the cardinal motor symptoms, as well as the manifestation of nonmotor symptoms including autonomic, sleep, sensory, gastrointestinal, oculomotor, and mood disturbances. ${ }^{6,7}$ In the advanced disease stages 25 to $40 \%$ of all PD patients display a dementing syndrome, usually referred to as PD with dementia (PDD). ${ }^{7}$ Most PD cases are idiopathic, although there are familial forms, several of which are monogenic and have a brain neuropathology more or less identical to that of idiopathic PD, for example, the alanine to proline (A30P) mutation of the alpha-synuclein protein. ${ }^{6,8}$

DLB represents the second most common dementing neurodegenerative disorder. ${ }^{3,9}$ The clinical manifestations include cardinal symptoms, that is, progressive and fluctuating dementia, recurrent visual hallucinations, and Parkinson-like motor manifestations and secondary symptoms (ie, autonomic, sleep, dysphagia, and oculomotor symptoms, depression, and executive dysfunction). ${ }^{3}$ As with PD, DLB cases are usually idiopathic, although several familial forms including monogenic causes are known. ${ }^{10}$

In the brains of PD and DLB patients, the following alpha-synuclein-immunopositive, insoluble aggregates occur: (1) Lewy bodies (LB) in the neuronal perikarya; (2) Lewy neurites (LN) in neuronal processes; (3) coiled bodies (CB) in oligodendrocytes. ${ }^{3,11-13}$ The PD-related brain pathology presumably propagates along neuronal interconnectivities in a prionlike manner and spreads in highly stereotypical temporal and topographical sequences, which were compiled into a neuropathological staging system by Braak et al. ${ }^{2,14}$ Because DLB patients exhibit similar brain distribution patterns of aggregation pathology as PD patients, it is tempting to suggest that analogous mechanisms of propagation are at work. ${ }^{12,15,16}$

Several of the motor symptoms of PD and DLB patients (ie, resting tremor, disturbed balance, gait and postural reflexes), do not respond well to the widely used L-dopa treatment but can be associated with pathological changes in pre- or postcerebellar circuits or the cerebellum itself, which was considered to be unaffected by the aggregation pathology in PD and DLB. ${ }^{2,17,18}$ Additionally, recent systematic studies demonstrated cerebellar

From the ${ }^{1}$ Dr. Senckenberg Chronomedical Institute, J. W. Goethe University, Frankfurt am Main, Germany; ${ }^{2}$ Luxembourg Center for Systems Biomedicine, University of Luxembourg, and Luxembourg Hospital Center, Luxembourg City, Luxembourg; ${ }^{3}$ Department of Neurology, Hertie Institute for Clinical Brain Research, and German Center for Neurodegenerative Diseases, Tübingen, Germany; and ${ }^{4}$ Department of Pathology and Medical Biology, University Medical Center Groningen, University of Groningen, Groningen, the Netherlands

Address correspondence to Dr Seidel, Dr. Senckenberg Chronomedical Institute, J. W. Goethe University, Frankfurt am Main, Germany. E-mail: Kay_Seidel@gmx.de

Additional supporting information can be found in the online version of this article.

Received Mar 23, 2016, and in revised form Apr 13, 2017. Accepted for publication Apr 15, 2017

View this article online at wileyonlinelibrary.com. DOI: 10.1002/ana 24937 

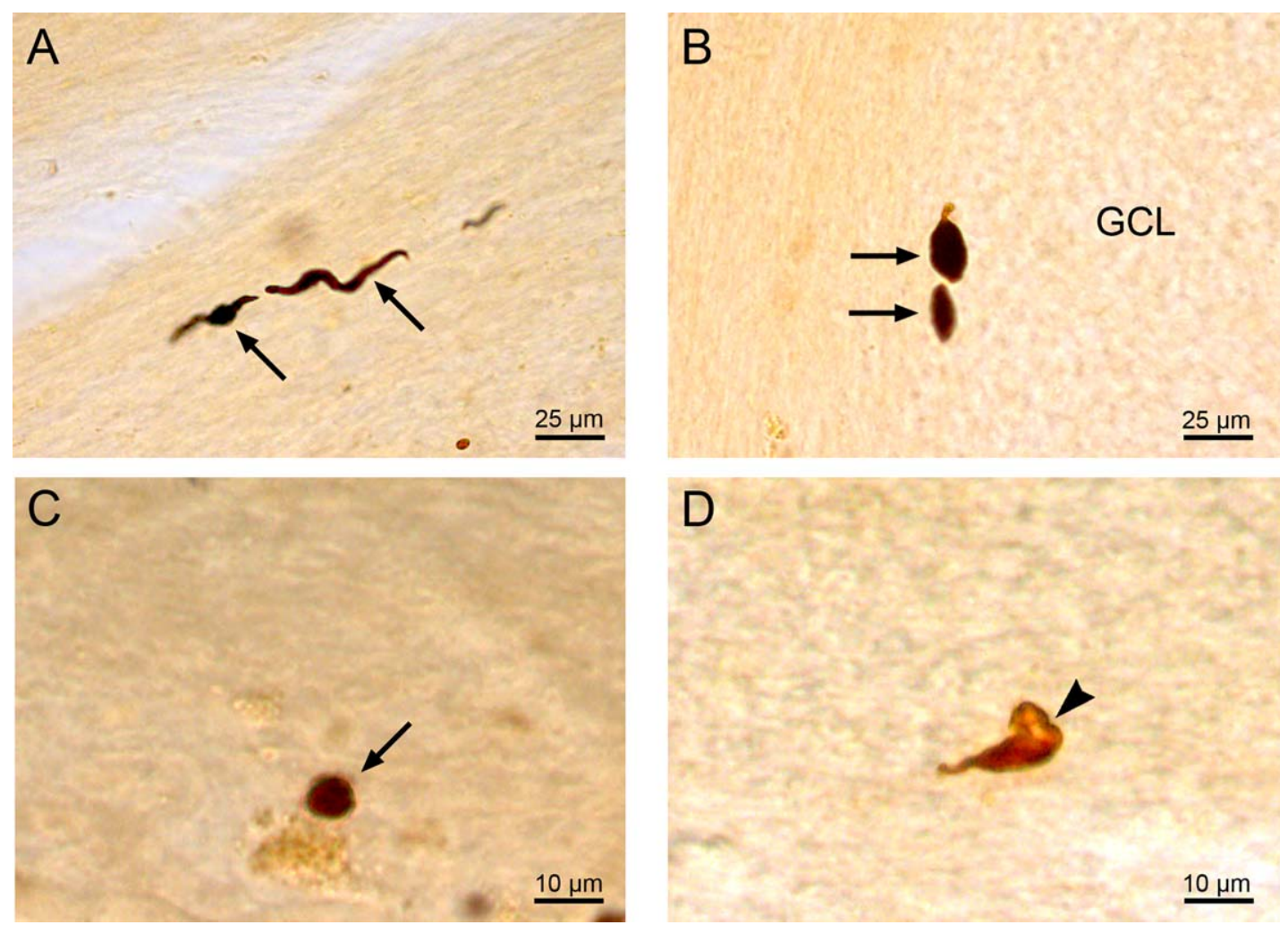

FIGURE 1: Types of cerebellar alpha-synuclein aggregates encountered in Parkinson disease and dementia with Lewy bodies. (A) A classical Lewy neurite (arrows) in the cerebellar white matter. (B) An ovoid Lewy neurite (arrows) in the white matter of a cerebellar lobule bordering on the granule cell layer (GCL). (C) A rare Lewy body (arrow) within a neuron of the fastigial nucleus. (D) Oligodendroglial coiled bodies (arrowhead) are frequently encountered within areas with marked or severe white matter pathology. (A-D: mouse anti-alpha-synuclein; $100 \mu \mathrm{m}$ thick, polyethylene glycol-embedded sections.) [Color figure can be viewed at www.annalsofneurology.org]

pathology in a monogenic PD patient with the A30P mutation as well as alpha-synuclein aggregates in precerebellar brainstem structures in idiopathic PD and DLB patients. ${ }^{8,12}$ This implies a distinct possibility that the cerebellum is affected by the aggregation pathology in idiopathic PD and DLB. Thus, we performed a systematic investigation of the neuropathology of the cerebella of PD and DLB patients and a reinvestigation of the cerebellum of a patient with the A30P mutation.

\section{Patients and Methods}

\section{Patients}

We examined the cerebella of $5 \mathrm{PD}, 1 \mathrm{PDD}$, and $5 \mathrm{DLB}$ patients as well as the cerebellum from a patient with the A30P mutation and 4 control cases (Supplementary Table 1). ${ }^{8}$ The study was approved by the Ethics Committee of the Faculty of Medicine at the Goethe University of Frankfurt am Main.

\section{Immunohistochemical Procedures}

The cerebella were fixed in $4 \%$ aqueous formalin, embedded in polyethylene glycol (PEG 1000; Merck, Darmstadt, Germany), and cut into sets of $100 \mu \mathrm{m}$-thick serial sagittal sections.

To assess neurodegeneration, 1 set of sections was treated with aldehyde-fuchsin and Darrow red (pigment-Nissl staining).
To visualize alpha-synuclein aggregation, a second set was immunostained with the monoclonal syn-1 antibody (catalog number 610786, 1:2,000; BD Biosciences, San Jose, CA), which targets amino acid 91-99 of rat alpha-synuclein (ATGFVKKDQ), a sequence that is $100 \%$ homologous to human alpha-synuclein. ${ }^{12}$

The severity of neuronal and oligodendroglial aggregation pathology in representative tissue sections through the deep cerebellar nuclei, the surrounding white matter, and the cerebellar lobules was scored as absent (0, cerebellar component devoid of aggregates), mild (1, cerebellar component with scarce aggregates), marked (2, cerebellar component with numerous aggregates), or severe (3, cerebellar component filled with aggregates). Medians were calculated for each area of interest. Neurodegenerative changes were evaluated by comparing pigment-Nissl-stained sections to matching control sections.

The nonparametric Friedman test was performed to document the reliability of our assessments, whenever $>1$ section was available for a given area of interest. We additionally confirmed our semiquantitative assessment of neurodegenerative changes by comparing the neuronal densities of 10 randomly chosen fields of vision within an area of interest between patient and control cerebella with the 2-sample $t$ test. All evaluations were performed using BIAS software for Windows. 

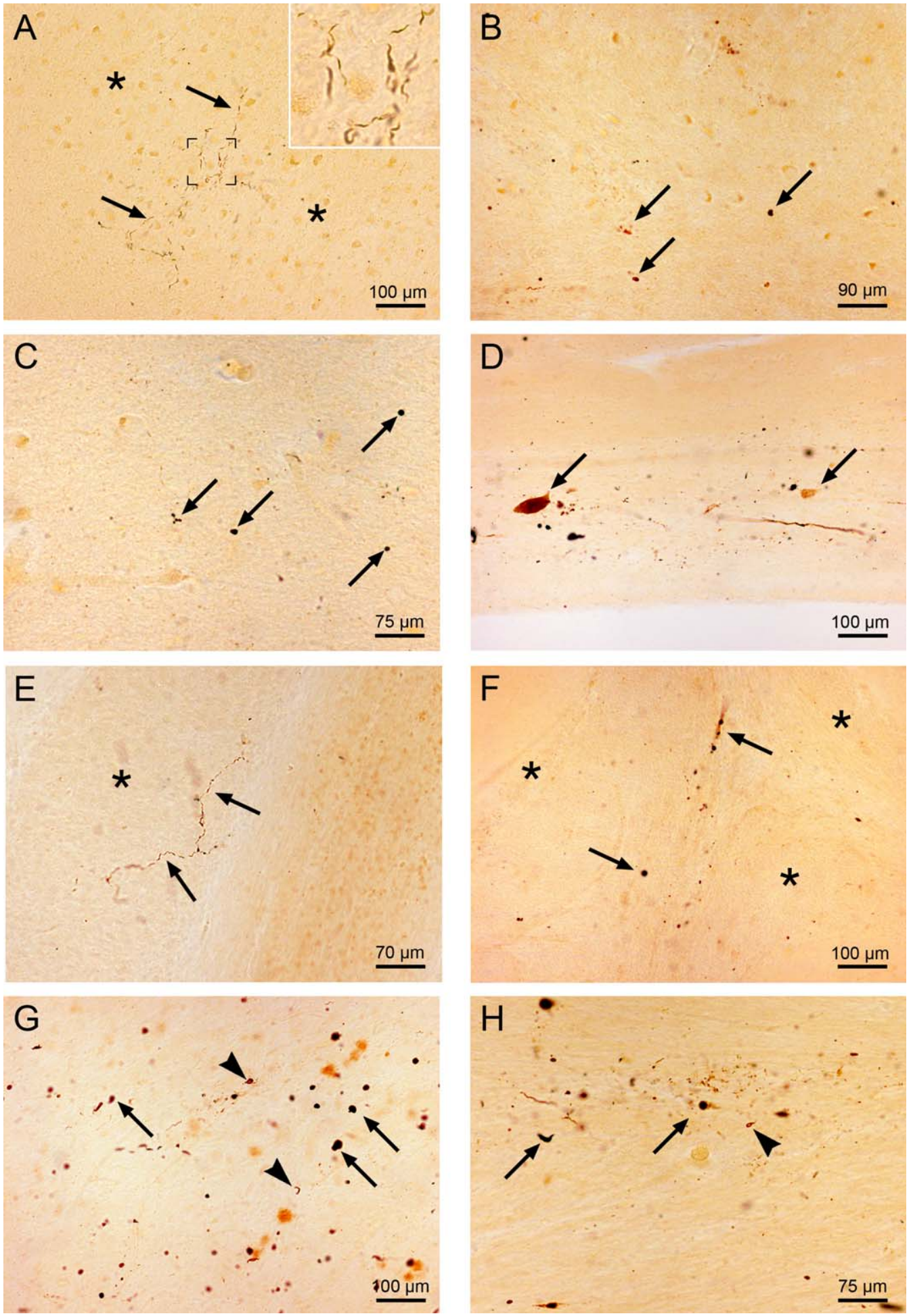

FIGURE 2: Alpha-synuclein aggregation in different cerebellar areas. (A) A meshwork of Lewy neurites in the dentate nucleus (arrows, insert), which is bordered by mostly unaffected areas (asterisks). (B, C) Marked alpha-synuclein pathology in the emboliform and fastigial nuclei, respectively (arrows). (D) Severe alpha-synuclein pathology in the pars cerebellaris locus coerulei (arrows). (E) A single affected branching neuron (arrows) within the otherwise unstained granular cell layer (asterisk). (F) Marked neuritic alpha-synuclein pathology (arrows) in the white matter of a cerebellar lobule, with mostly unstained neighboring granular layer regions (asterisks). (G, H) Severe alpha-synuclein pathology (arrows), including several coiled bodies (arrowheads) in the white matter surrounding the deep cerebellar gray matter. (A-H: mouse anti-alpha-synuclein; $100 \mu \mathrm{m}$ thick, polyethylene glycol-embedded sections.). [Color figure can be viewed at www.annalsofneurology.org] 
TABLE 1. Severity of Aggregation Pathology Scored Separately for LP and Frequency of CB

\begin{tabular}{|c|c|c|c|c|c|c|c|}
\hline Patient & Deep White & $\begin{array}{l}\text { Fastigial } \\
\text { Nuclei }\end{array}$ & $\begin{array}{l}\text { Globose } \\
\text { Nuclei }\end{array}$ & $\begin{array}{l}\text { Emboliform } \\
\text { Nuclei }\end{array}$ & $\begin{array}{l}\text { Dentate } \\
\text { Nuclei }\end{array}$ & PCLC & Lobules \\
\hline \multicolumn{8}{|l|}{1} \\
\hline LP & 2 & 1 & 2 & 1 & 1 & 2 & $1(0-2)$ \\
\hline CB & $1(0-1)$ & 0 & 0 & 0 & 1 & 1 & $0(0-1)$ \\
\hline \multicolumn{8}{|l|}{2} \\
\hline LP & 2 & 1 & $2(1-2)$ & 2 & 2 & 3 & $1(1-2)$ \\
\hline CB & 1 & $0(0-1)$ & 1 & 0 & 1 & 2 & $0(0-1)$ \\
\hline \multicolumn{8}{|l|}{3} \\
\hline LP & 2 & 2 & 2 & 2 & 2 & $3(2-3)$ & $1.5(1-2)$ \\
\hline $\mathrm{CB}$ & $2(1-2)$ & 1 & 1 & 1 & 1 & 1 & $1(0-2)$ \\
\hline \multicolumn{8}{|l|}{4} \\
\hline LP & $2(1-2)$ & $0.5(0-1)$ & 1 & 1 & 2 & 2 & $1(1-2)$ \\
\hline $\mathrm{CB}$ & 1 & $0.5(0-1)$ & 0 & 0 & 0 & 1 & $0(0-1)$ \\
\hline
\end{tabular}

5

$\begin{array}{llllllll}\text { LP } & 2(1-2) & 1 & 1 & / & 1 & / & 1(1-2) \\ \text { CB } & 2(1-2) & 1 & 0 & / & 1(0-1) & / & 0(0-1)\end{array}$

6

\begin{tabular}{|clllllll}
\hline LP & 2 & 1 & 2 & 2 & 2 & 3 & $1(1-2)$ \\
\hline CB & 2 & 1 & 2 & 1 & 1 & 1 & $1(0-2)$ \\
\hline 7 & & & & & \\
\hline LP & $2(2-3)$ & 2 & 2 & 2 & 2 & 3 & $2(2-3)$ \\
\hline CB & 2 & 1 & 1 & 1 & 1 & 1 & $2(1-2)$ \\
8 & & & & & & \\
\hline LP & $2(1-2)$ & 2 & 1 & $1.5(1-2)$ & 1 & 2 & $1(0-2)$ \\
CB & $1(0-1)$ & $0.5(0-1)$ & $0.5(0-1)$ & $0.5(0-1)$ & 0 & 0 & $0(0-1)$ \\
\hline
\end{tabular}

9

$\begin{array}{llllllll}\text { LP } & 2 & 1 & 1 & 2 & 2 & 3(2-3) & 2(1-2) \\ \text { CB } & 1(1-2) & 1 & 1 & 1 & 1 & 1 & 1(0-2)\end{array}$

10

$\begin{array}{llllllll}\text { LP } & 2 & 2 & 2 & 2 & 2 & 3 & 1(1-2) \\ \text { CB } & 1 & 1 & 0.5(0-1) & 1 & 1 & 1 & 0(0-1)\end{array}$

11

$\begin{array}{llllllll}\mathrm{LP} & 3 & 1 & 1.5(1-2) & 2 & 2 & 3(2-3) & 2(1-3) \\ \mathrm{CB} & 2 & 1 & 1 & 1 & 1 & 1(1-2) & 0(0-2)\end{array}$

12

$\begin{array}{llllllll}\text { LP } & 2(2-3) & 1.5(1-2) & 1 & 2 & 2 & 3 & 2(1-2) \\ \text { CB } & 1(1-2) & 1 & 0 & 1 & 1 & 1 & 0(0-1)\end{array}$

Areas investigated are: deep white matter; fastigial, globose, emboliform, and dentate nuclei; PCLC; and cerebellar lobules. The severities are scored as 0 , unaffected; 1 , mildly affected; 2 , moderately affected; 3 , severely affected. / = Section unavailable. When cerebellar areas were assessed in $>1$ slide in a single patient and for all cerebellar lobules, medians and ranges are denoted.

$\mathrm{CB}=$ coiled bodies; LP = Lewy body/neurite pathology; PCLC $=$ pars cerebellaris locus coerulei. 


\section{Results}

The cerebella of all patients displayed neuronal and oligodendroglial alpha-synuclein aggregation pathology, with no significant differences of severity between the disease groups (Fig 1). The neuronal aggregation pathology consisted predominantly of LN, whereas LB were significantly rarer (Fig 2). Alpha-synuclein-immunopositive oligodendroglial inclusions were frequently present at cerebellar sites with at least marked neuronal aggregation pathology (see Fig 2, Table). No alpha-synuclein-immunopositive aggregates were detectable in control cerebella.

The central cerebellar nuclei (ie, fastigial, globose, emboliform, and dentate nuclei) exhibited mild to marked aggregation pathology, showing mostly LN in associated neurites and a few LB (see Fig 2, Table). The pars cerebellaris locus coerulei (PCLC) consistently displayed marked aggregation pathology, with an abundance of $\mathrm{LN}, \mathrm{LB}$, and $\mathrm{CB}$ that was comparable to the brainstem portion of the locus coeruleus. ${ }^{12,19}$

The deep cerebellar white matter exhibited severe aggregation pathology. It consisted almost exclusively of elongated or ovoid LN, located adjacent to the cerebellar peduncles, in the deep cerebellar nuclei, and in the neuropil neighboring the PCLC (see Fig 2). The neuronal protein aggregates were frequently accompanied by alpha-synuclein-immunopositive CB.

The cerebellar cortex showed only minor involvement (see Table 1, Supplementary Table 2). In the molecular, Purkinje, and granular cell layers, alphasynuclein-immunopositive aggregates occurred only occasionally, whereas they were more frequent in the lobular white matter tracts.

The Friedman test showed a significant difference between sections in only 1 of 38 investigated groupings. There was no statistically significant difference between the aggregation pathology of PD and DLB cases. The cerebella showed no visible neurodegeneration, with no significant differences in neuronal density between matching controls (Patients 15 and 16) and patients.

\section{Discussion}

For a long time, the cerebellum was considered to be unaffected in the synucleinopathies PD and DLB, and was not incorporated in the Braak staging model. ${ }^{2,3,17}$ However, the inclusion pathology of precerebellar brainstem nuclei and fiber tracts points to a possible involvement of the cerebellum in the alpha-synuclein aggregation pathology. ${ }^{12,17}$ In the present study, we could for the first time demonstrate the consistent presence of alpha-synuclein-immunopositive neuronal and oligodendroglial inclusions as a pathological alteration in the cerebellum of idiopathic PD and DLB patients, with moderate to severe aggregation pathology at the deep cerebellar nuclei and neighboring white matter tracts and a milder involvement of the lobules. This new discovery of cerebellar inclusion pathology was facilitated by the use of $100 \mu$ m-thick PEG-embedded tissue sections, which, due to the superposition of neuroanatomical structures, allowed the recognition of rare alpha-synuclein aggregations (eg, in the cerebellar lobules). The highly similar distribution of this cerebellar pathology in both patient groups studied underscores that PD and DLB not only share many clinical symptoms, but are also neuropathologically indistinguishable in advanced disease stages and actually represent different points on the same clinicopathological spectrum. ${ }^{4,5}$

Currently it is widely held that the alpha-synuclein pathology of PD and DLB propagates transneuronally along neuroanatomical connections in a prionlike manner. ${ }^{14,20}$ This concept is supported by: (1) the typical distribution pattern of the PD-related inclusion pathology, (2) experimental disease models demonstrating neuron-to-neuron transmission of pathologically altered alpha-synuclein, and (3) the invasion of neuronal grafts in PD patients by the aggregation pathology. Our own findings, together with the known involvement of precerebellar brainstem nuclei and the ventrolateral thalamic nucleus, which receives projections from the cerebellum, support this notion. ${ }^{12}$

The clinical manifestation of tremor, gait impairment, and postural instability, and the weak response of these symptoms to L-dopa therapy, suggest that these symptoms are not primarily caused by the involvement of the basal gangliothalamocortical loop. ${ }^{17,18}$ Although the demonstrated aggregation pathology was only moderate, and co-occurring neurodegeneration was not discernable, the hypothesis that these pathological changes contribute to tremor and disturbed balance, gait, and postural reflexes cannot be excluded, and could be investigated in functional imaging studies. ${ }^{13}$ Interestingly, the deep cerebellar pathology was often more severe than the alpha-synuclein pathology in most precerebellar structures. ${ }^{12}$

Although we could for the first time demonstrate alpha-synuclein inclusion pathology within the cerebellum in PD and DLB, the time point at which the aggregation pathology manifests remains to be elucidated. As the affection of the cerebellum has not been considered in PD and DLB staging systems, a reinvestigation of PD- and DLB-related neuropathology regarding cerebellar and precerebellar structures in a larger patient cohort, including early cases, would be warranted.

\section{Acknowledgment}

This study was supported by grants from the Senckenberg Foundation. 
We thank D. von Meltzer for skillful secretarial assistance.

\section{Author Contributions}

K.S. und U.R. contributed to the design of the study. K.S., R.K., L.S., W.F.A.d.D., H.-W.K., and U.R. contributed to the drafting of the manuscript. K.S., M.B., and N.H. contributed to data acquisition.

\section{Potential Conflicts of Interest}

Nothing to report.

\section{References}

1. Jellinger KA. Neuropathological spectrum of synucleinopathies. Mov Disord 2003;18(suppl 6):S2-S12.

2. Braak H, Del Tredici K, Rub U, et al. Staging of brain pathology related to sporadic Parkinson's disease. Neurobiol Aging 2003;24:197-211.

3. McKeith IG, Dickson DW, Lowe J, et al. Diagnosis and management of dementia with Lewy bodies: third report of the DLB Consortium. Neurology 2005;65:1863-1872.

4. Chartier-Harlin MC, Kachergus J, Roumier C, et al. Alpha-synuclein locus duplication as a cause of familial Parkinson's disease. Lancet 2004;364:1167-1169.

5. Singleton $A B$, Farrer $M$, Johnson J, et al. alpha-Synuclein locus triplication causes Parkinson's disease. Science 2003;302:841.

6. de Lau LM, Breteler MM. Epidemiology of Parkinson's disease. Lancet Neurol 2006;5:525-535.

7. Gelb DJ, Oliver E, Gilman S. Diagnostic criteria for Parkinson disease. Arch Neurol 1999;56:33-39.

8. Seidel K, Schols L, Nuber S, et al. First appraisal of brain pathology owing to A30P mutant alpha-synuclein. Ann Neurol 2010;67:684-689.
9. Zaccai J, McCracken C, Brayne C. A systematic review of prevalence and incidence studies of dementia with Lewy bodies. Age Ageing 2005;34:561-566

10. Meeus B, Theuns J, Van Broeckhoven C. The genetics of dementia with Lewy bodies: what are we missing? Arch Neurol 2012;69: 1113-1118

11. Wakabayashi K, Hayashi S, Yoshimoto M, et al. NACP/alpha-synuclein-positive filamentous inclusions in astrocytes and oligodendrocytes of Parkinson's disease brains. Acta Neuropathol 2000;99: $14-20$

12. Seidel K, Mahlke J, Siswanto S, et al. The brainstem pathologies of Parkinson's disease and dementia with Lewy bodies. Brain Pathol 2015;25:121-135.

13. Volpicelli-Daley LA, Luk KC, Patel TP, et al. Exogenous alphasynuclein fibrils induce Lewy body pathology leading to synaptic dysfunction and neuron death. Neuron 2011;72:57-71.

14. Angot E, Steiner JA, Hansen C, et al. Are synucleinopathies prionlike disorders? Lancet Neurol 2010;9:1128-1138.

15. Jellinger KA. Significance of brain lesions in Parkinson disease dementia and Lewy body dementia. Front Neurol Neurosci 2009 24:114-125.

16. Grinberg LT, Rueb U, Alho AT, et al. Brainstem pathology and non-motor symptoms in PD. J Neurol Sci 2010;289:81-88.

17. Wu T, Hallett M. The cerebellum in Parkinson's disease. Brain 2013;136:696-709

18. Paulus $W$, Jellinger $K$. The neuropathologic basis of different clinical subgroups of Parkinson's disease. J Neuropathol Exp Neurol 1991;50:743-755.

19. Braak H. On the pars cerebellaris loci coerulei within the cerebellum of man. Cell Tissue Res 1975;160:279-282.

20. Hansen C, Li JY. Beyond alpha-synuclein transfer: pathology propagation in Parkinson's disease. Trends Mol Med 2012;18: 248-255. 